\title{
Multiple athletic performances, maturation, and Functional Movement Screen total and individual scores across different age categories in young soccer players
}

\author{
Igor Bakalár, Jaromír Šimonek, Janka Kanásová, Bohumila Krčmárová, Matúš Krčmár* \\ Department of Physical Education and Sports, Faculty of Education, Constantine the Philosopher University in Nitra, Nitra, Slovakia
}

The purpose of this study was to evaluate differences in multiple athletic performances, and to examine associations between athletic performance and Functional Movement Screen (FMS) in young soccer players. Forty-one soccer players were tested on peak height velocity (PHV), 5-, 10-, and 30-m sprint times, 505 change of direction (505 COD), Y-reactive agility tests, countermovement jump (CMJ), and squat jump (SJ) height. Significant main effects $(P<0.01)$ were recorded in all tests except FMS total score whereas the U16 group outperformed U12 and U14 in almost each test. However, when the results were adjusted to the PHV 7 of 11 tests were nonsignificant. Significant associations were recorded between trunk stability push-up (TSPU) and $5 \mathrm{~m}(P=0.04)$ and
505 COD (preferred) times $(P=0.01)$, and SJ height $(P=0.03)$ in the U12. In the U14, significant associations were recoded between TSPU and SJ $(P<0.01)$ and CMJ height $(P=0.03)$. In the U16, significant associations were recorded between deep overhead squat and 5-m sprint time $(P=0.02)$ and CMJ height $(P=0.04)$. Results of this study indicate that athletic performance in young soccer players is multidimensional in nature, and it is a consequence of several factors including maturation, different training strategies, and movement proficiency.

Keywords: Youth soccer, Adolescence, Physical testing, Movement screening

\section{INTRODUCTION}

Muscular strength, linear sprinting, sprinting with change of direction and agility as a reaction to external stimuli are important components of well-designed strength and conditioning program that can affect athletic performance in adult (Loturco et al., 2016) as well as young soccer players (Kobal et al., 2017). Despite the training methods that are frequently used in older and more experienced soccer players copying this approach in young players seems to be inappropriate mainly due to the limitations related to maturation (Behm et al., 2008). Few studies examined physical performance across different age categories in young soccer players in relation to the maturation process (Lloyd et al., 2015; MendezVillanueva et al., 2011). In both studies, the oldest group had always achieved the better performance in various tests (e.g., acceleration, maximal running speed, squat jump), however, when the results were adjusted to peak height velocity almost each significance disappeared (Lloyd et al., 2015; Mendez-Villanueva et al., 2011). The results suggest that players who are advanced in biological maturation may have better performance results in muscular strength and power, running speed as well as endurance compared to the less matured peers (Figueiredo et al., 2009; Malina et al., 2004). Despite widespread physical performance testing across age categories in young as well as adult soccer players, injury prevention program and/or screening has become the standard procedure within the test battery.

One of the methods that is widely accepted among numerous coaches, researchers, and various educational institutions and universities in the world is the Functional Movement Screen testing (FMS). FMS was invented by Gray Cook and Lee Burton and at a basic level, it contains seven screening tests that assess several areas (Cook, 2011). The seven basic tests include: deep overhead
*Corresponding author: Matúš Krčmár (D) https://orcid.org/0000-0003-1538-6631 Department of Physical Education and Sports, Faculty of Education, Constantine the Philosopher University in Nitra, Tr. A. Hlinku 1, 94974 Nitra, Slovakia E-mail:krcmo300@gmail.com

Received: July 17, 2020 / Accepted: August 15, 2020
This is an Open Access article distributed under the terms of the Creative Commons Attribution Non-Commercial License (https://creativecommons.org/licenses/by-nc/4.0/) which permits unrestricted non-commercial use, distribution, and reproduction in any medium, provided the original work is properly cited. 
squat (DS), hurdle step (HS), in-line lunge (ILL), shoulder mobility (SM), active straight leg raise (ASLR), trunk stability push-up (TSPU), rotary stability (RS) (Cook, 2011). Several research studies examined the FMS in relation to injury prevention (Kiesel et al., 2007; Letafatkar et al., 2014; Newton et al., 2017) and different performance characteristics (Lee et al., 2019; Lloyd et al., 2015; Lockie et al., 2015; Parchman and McBride, 2011). Among the mentioned studies (besides the others) a considerable part of the studies has been done on soccer players including prestigious soccer academies and clubs (Marques et al., 2017; McCall et al., 2015; Newton et al., 2017). However, it is not surprising that the best clubs or academies use this screening tool in soccer as it offers affordable and relatively quick tool that can be used to assess potential movement deficiencies in players (Bardenett et al., 2015).

Despite of the abovementioned studies and current trends, several studies tried to examine potential sensitivity of the FMS to predict injuries and establish associations to multiple performance characteristics. For instance, Newton et al. (2017) examined injury occurrence in relation to the FMS score in youth soccer players. Their findings suggest that no relationship exists between achieved scores and injury. Similarly, Smith and Hanlon (2017) found no significant difference between FMS, and noncontact injuries in soccer players. In regards of potential association of the FMS to performance characteristics, several studies found significant associations of either total score or subset of the FMS tests in soccer players (Lee et al., 2019; Lloyd et al., 2015; Silva et al., 2017) but some did not (Parchmann and McBride, 2011; Zalai et al., 2015), however the study by Parchman and McBride (2011) was done on golfers. Collectively, from the results of the abovementioned studies, it seems that individual scores are more able to detect potential associations between the FMS, and athletic performances (Silva et al., 2017) which could be explained by different subtests that assess several areas not all of which may be performance related. Also, besides the ability of the FMS to potentially predict noncontact injuries which according to the mentioned studies are in an agreement, however, the opposite may hold true, when examining association to the physical abilities with contrast findings in youth players, whereas the differences may be caused as a result of combination of both the movement quality, and growth and/or maturation.

Therefore, the purpose of this study was twofold. At the first, to assess differences between physical characteristics in young soccer players of different age, and secondly, to assess the FMS scores and multiple physical characteristics in youth soccer categories, and thus to detect potential performance-related associations.

\section{MATERIALS AND METHODS}

\section{Experimental design}

The present study represents cross-sectional design. All players who met inclusion criteria across different age categories were tested using the FMS method and performed physical tests assessment in a period of 4 days during the end of the actual season (November, 2019).

\section{Participants}

In total, 41 soccer players met inclusion criteria and were included into this study. Participants were divided into three groups (U12, U14, U16) according to the age category they play (Table 1). All research procedures were explained to players, coaches as well as parents. Informed consent was signed by coaches and parents as well. The study was approved by local soccer academy and University Ethic Committee (registration number: V/2/2019) to comply with the Declaration of Helsinki.

\section{Procedures}

Participants underwent four testing sessions. During the first and the second session, they performed FMS testing (half of the participants on session 1 and the other half on session 2). During the next 2 sessions, they performed physical performance testing which included 5-, 10-, and 30-m sprint time, 505 change of direction test (505 COD), Y-reactive agility test (Y-test), squat jump (SJ), and countermovement vertical jump (CMJ) height. During the 505 COD test participants changed direction to the left and to the right side or to preferred and nonpreferred direction. COD deficit as difference between 505 COD time and 10-m linear sprinting time was also calculated in each group. The reason why testing sessions were divided into four ones lies in the fact that all tests were time consuming, especially the FMS testing, and there was only limited time opportunity when all the participants could attend testing sessions.

Biological maturity was assessed noninvasively by measuring standing height $(\mathrm{cm})$, sitting height $(\mathrm{cm})$, and body weight $(\mathrm{kg})$

Table 1. Basic characteristics of players across age groups

\begin{tabular}{|c|c|c|c|c|c|}
\hline Group & Age (yr) & $\begin{array}{c}\text { Standing } \\
\text { height }(\mathrm{cm})\end{array}$ & $\begin{array}{c}\text { Sitting } \\
\text { height }(\mathrm{cm})\end{array}$ & $\begin{array}{l}\text { Body weight } \\
(\mathrm{kg})\end{array}$ & $\begin{array}{c}\text { Peak height } \\
\text { velocity }\end{array}$ \\
\hline $\mathrm{U} 12(\mathrm{n}=15)$ & 11 & $146.5 \pm 6.0$ & $76.9 \pm 3.9$ & $39.0 \pm 6.3$ & $-2.2 \pm 0.5$ \\
\hline U14 (n=14) & 13 & $162.4 \pm 9.4$ & $86.2 \pm 4.7$ & $49.2 \pm 6.6$ & $-0.1 \pm 0.7$ \\
\hline U16 $(n=12)$ & 15 & $175.2 \pm 4.3$ & $93.0 \pm 2.4$ & $62.6 \pm 2.1$ & $1.9 \pm 0.4$ \\
\hline
\end{tabular}

Values are presented as mean \pm standard deviation. 
during the time of the physical performance testing. From these measures peak height velocity was calculated (Mirwald et al., 2002) which represents the predicted period of time in which a child grows at the fastest rate (Lloyd and Oliver, 2012).

The FMS testing was carried out by two certified testers and certified strength and conditioning specialist with at least with 2 years of FMS screening experience with the participation of the local soccer club physiotherapist. Seven basic tests were performed in the following order: (a) DS, (b) HS, (c) ILL, (d) SM, (e) ASLR, (f) TSPU, (g) RS (Fig. 1). As there was no zero value obtained during testing, no additional coding was required (in case of value " 0 "). Briefly, the point scale ranged from 1 to 3 points where 1 represents situation of unable to perform required pattern, 2 situations with correct pattern performed but with compensation, and 3 points
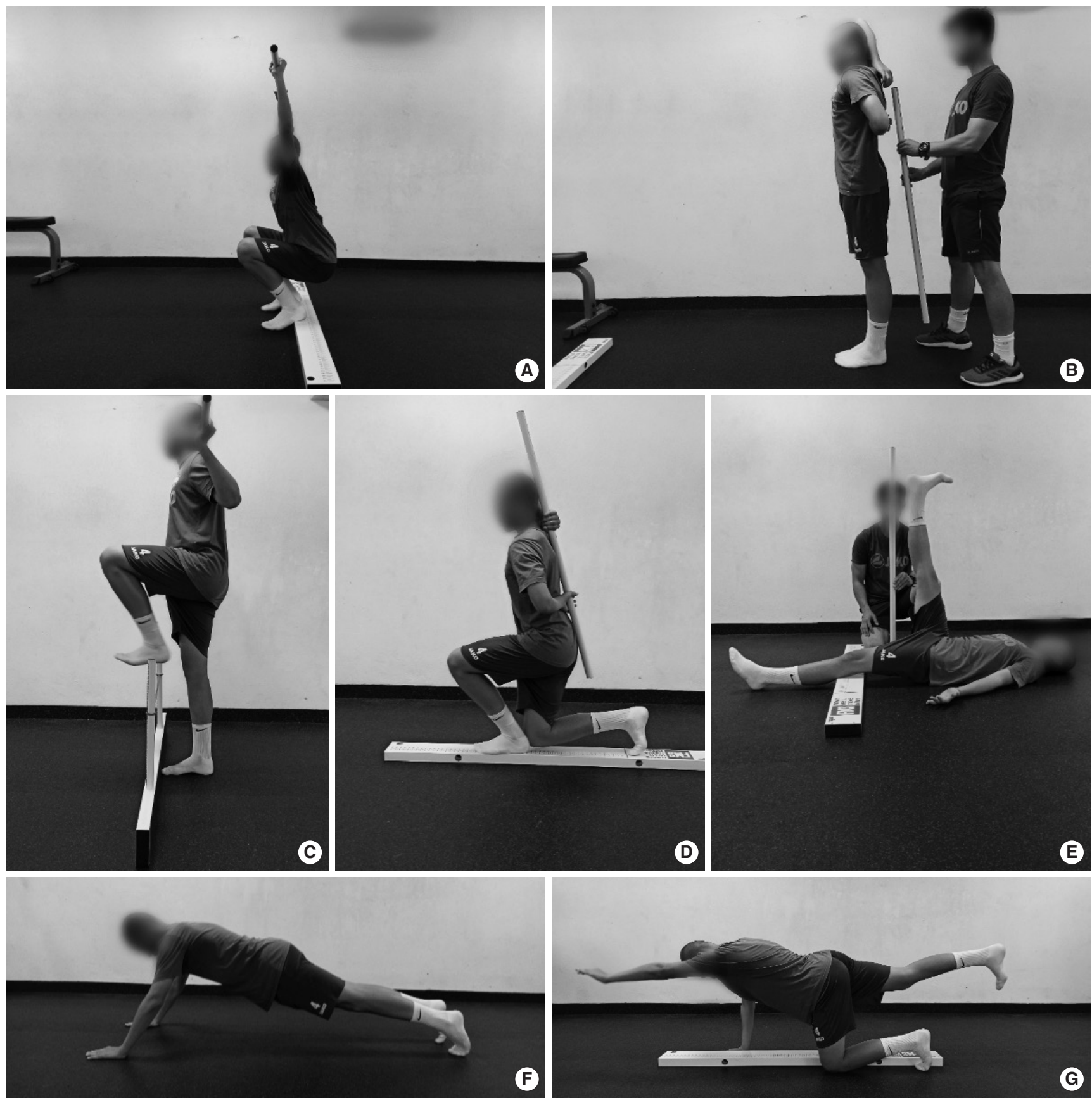

E

Fig. 1. Testing of the Functional Movement Screen. 
represented correct pattern as directed. All the testing was done in accordance with the FMS methodology (for more details see: Cook, 2011). Total score represented the sum of individual subtests. The FMS shows good test-retest reliability (Interclass correlation coefficient [ICC], 0.6) and excellent reliability (ICC, 0.92) for the liveversus-video session (Shultz et al., 2013).

Sprints over 5-, 10-, and 30-m dash, 505 COD test, and Y-test were recorded using Witty wireless training timer (Microgate, Bolzano, Italy). All sprints were performed in one run and measured as split times (5-10-30-m). Participants had three trials from which the best time was recorded for further analysis. They started from stationary standing position with the dominant foot placed behind the starting line. In 505 COD test, they run and changed direction on the dominant and nondominant leg (preferred/nonpreferred leg) with two trials on each side with the best time chosen for further analysis (Fig. 2). Y-reactive agility test was also performed with Witty system but in this case, additional equipment (WittySEM, Bolzano, Italy) was added to the basic set-up to ensure video stimuli in the form of arrows indicating direction of the subsequent sprint after display (Fig. 3). Three trials were allowed to participants in the Y-reactive agility test with the best attempt for further analysis. Stimuli were displayed with following a 0.5 -sec de-

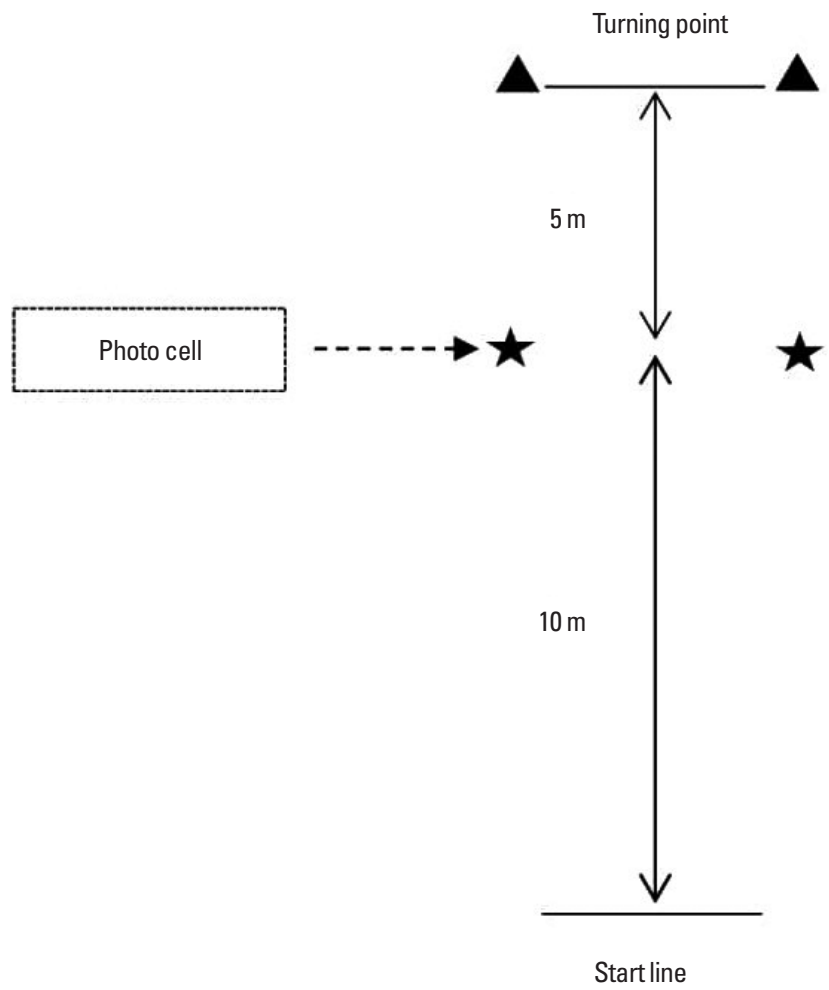

Fig. 2. 505 change of direction test. lay after triggering the second timing gate. COD deficit was calculated as mean 505 time (nonpreferred/preferred) - mean 10 sprint time. In overall, rest interval between trials was 2-3 minutes in all tests. Participants were instructed and encouraged to "run as fast as possible" from the beginning to the end of the dash.

Squat jump and countermovement jump height was recorded using Optojump Next (Microgate, Bolzano, Italy). Participants had three attempts in both variations with at least 30-sec rest between jumps. In both variations, participants were asked to put their hands on the hips and hold them there during the whole demonstration of the jumps. The highest vertical jump height was recorded and used for further analysis. Participants were encouraged to "jump as high as possible."

Test-retest reliability of our tests from our laboratory is shown in Table 2. In 505 COD test, test-retest reliability was examined elsewhere (ICC ranged from 0.84 to 0.89 with coeficient of variation from $1.6 \%$ to $1.8 \%$ ) and this was also done in youth soccer players in different age categories U11-U17 with the same device as used in our study (Dugdale et al., 2020).

\section{Statistical analyses}

Normality of distribution in each category was performed by

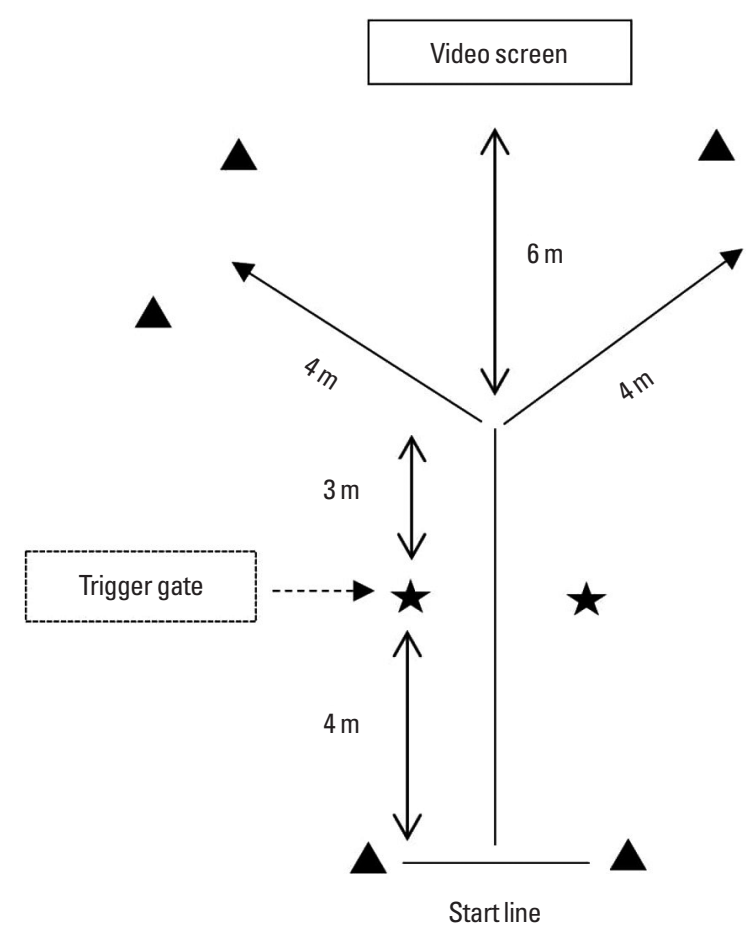

Fig. 3. Y-reactive agility test. 
Table 2. Interclass correlation coefficients (ICCs) with 95\% confidence intervals (95\% Cl)

\begin{tabular}{lc}
\hline Variable & ICC (95\% Cl) \\
\hline $5 \mathrm{~m}(\mathrm{sec})$ & $0.97(0.83-0.98)$ \\
$10 \mathrm{~m}(\mathrm{sec})$ & $0.99(0.95-0.98)$ \\
$30 \mathrm{~m}(\mathrm{sec})$ & $0.98(0.95-0.99)$ \\
Y-test (sec) & $0.82(0.39-0.95)$ \\
Squat jump (cm) & $0.99(0.94-0.99)$ \\
Countermovement jump (cm) & $0.97(0.91-0.99)$ \\
\hline
\end{tabular}

Shapiro-Wilk test. All data in each group showed normal distribution. Comparison of physical performance and FMS total score between groups was performed by 1-way analysis of variance (ANOVA) with Bonferroni post hoc test. Pearson product-moment correlation was performed to detect association between physical performances and FMS total score in each category. Spearman rank correlation coefficient was also calculated to obtain association between physical performance and individual subtests of the FMS in each category. Strength of the associations according to the Hopkins (2016) are: $0.00-0.09$ trivial; $0.10-0.29$ small; $0.30-0.50$ moderate; $0.51-0.70$ large; $0.71-0.90$ very large; $0.91-0.99$ nearly perfect; 1.00 perfect. Kruskal-Wallis H-test (nonparametric rank-based alternative of ANOVA) with Mann-Whitney $U$-test as post hoc test was used to calculate differences between the groups in each subtest of the FMS. Additionally, multivariate analysis of covariance was performed to analyse differences between performance characteristics adjusted to PHV as a covariate. Effect size was calculated according to the Hedges $g$, where $<0.49$ is a small effect, $0.50-0.79$ is a moderate effect, and $\geq 0.80$ is a large effect. 95\% confidence intervals are also presented in the text. All analyses were performed using IBM SPSS Statistics ver. 21.0 (IBM Co., Armonk, NY, USA).

\section{RESULTS}

ICCs with 95\% confidence intervals are shown in Table 1. Results of the study indicate that older players significantly outperformed younger ones in almost each examined measure except FMS total score where no significant differences between the groups were detected (Table 3). Additionally, right part of the table indicates results based on the PHV adjustment as a covariate.

The U16 group was significantly quicker compared to the U12 $(P<0.01,-0.15$ to $-0.04 \mathrm{sec})$ and $\mathrm{U} 14(P<0.01,-0.14$ to -0.03 $\mathrm{sec})$ on $5-\mathrm{m}$ dash. The same results were also obtained on the $10-\mathrm{m}$ and 30-m dash. In the COD 505 test, U14 and U16 were signifi-
Table 3. Results of 1-way ANOVA and multivariate ANCOVA with peak height velocity as a covariate between the groups in each test

\begin{tabular}{lcccccc}
\hline \multirow{2}{*}{ Performance } & \multicolumn{2}{c}{ ANOVA } & & \multicolumn{2}{c}{ ANCOVA } \\
\cline { 2 - 3 } \cline { 6 - 7 } & Ftest & & $P$-value & & Ftest & $P$-value \\
\hline $5 \mathrm{~m}$ (sec) & 11.7 & $<0.01$ & & 2.7 & $>0.05$ \\
$10 \mathrm{~m}(\mathrm{sec})$ & 17.1 & $<0.01$ & & 2.6 & $>0.05$ \\
$30 \mathrm{~m}$ (sec) & 46.9 & $<0.01$ & & 6.2 & $<0.01$ \\
$505 \mathrm{COD}$ (non) (sec) & 12.8 & $<0.01$ & & 1.2 & $>0.05$ \\
$505 C O D$ (pref) (sec) & 22.9 & $<0.01$ & & 1.1 & $>0.05$ \\
CODdef (pref) (sec) & 4.7 & $<0.05$ & & 2.6 & $>0.05$ \\
CODdef (non) (sec) & 7.0 & $<0.01$ & & 3.2 & $>0.05$ \\
Y-test (sec) & 49.6 & $<0.01$ & & 6.5 & $<0.01$ \\
Countermovement jump (cm) & 20.2 & $<0.01$ & & 5.8 & $<0.01$ \\
Squat jump (cm) & 18.7 & $<0.01$ & & 7.1 & $<0.01$ \\
FMS TS & 2.8 & 0.06 & & 0.8 & $>0.05$
\end{tabular}

ANOVA, analysis of variance; ANCOVA, analysis of covariance; 505 COD (non/pref), 505 change of direction test with nonpreferred/preferred turning leg; CODdef (non/ pref), change of direction deficit with nonpreferred/preferred turning leg; Y-test, Y-reactive agility test; FMS TS, Functional Movement System-total score.

cantly quicker compared to the $\mathrm{U} 12(P<0.01)$ (in both variations). In the CODdeficit (preferred), U16 and U14 achieved significantly better result compared to the U12 $(P<0.05)$, and in the CODdeficit (nonpreferred), U14 and U16 achieved significantly better results compared to the $\mathrm{U} 12(P<0.01$ and $P<0.05$, respectively). In the Y-test, the U16 significantly outperformed U14 $(P<0.01$, -0.51 to $-0.24 \mathrm{sec})$ and $\mathrm{U} 12$ as well $(P<0.01,-0.63$ to $-0.37 \mathrm{sec})$. The U14 was also significantly quicker compared to the U12 $(P<0.05,-0.25$ to $-0.003 \mathrm{sec})$. In the CMJ U16 jumped significantly higher compared to the U12 $(P<0.01,4.57$ to $11.93 \mathrm{~cm})$ and U14 $(P<0.01,4.56$ to $12.03 \mathrm{~cm})$. Similarly in the SJ U16 jumped significantly higher compared to the U12 $(P<0.01,3.75$ to $11.0 \mathrm{~cm})$ and $\mathrm{U} 14(P<0.01,4.57$ to $11.93 \mathrm{~cm})$. When comparing individual FMS subtests, only significant main effect was recorded in the TSPU $(P<0.01)$. Post hoc comparison revealed that both U14 and U16 achieved significantly higher score compared to the U12 (U14 vs. U12: $z=-2.49, P=0.02 ; \mathrm{U} 16$ vs U12: $z=-3.41$, $P<0.01)$

Interestingly, when performance characteristics were adjusted to the PHV as a covariate, 7 of 11 tests were nonsignificant $(P>$ $0.05)(5 \mathrm{~m}, 10 \mathrm{~m}$, both COD variations as well as COD deficits and FMS total score). Significance persisted in the $30 \mathrm{~m}$ and Y-reactive agility sprint times, $\mathrm{CMJ}$, and $\mathrm{SJ}$ height $(P<0.01)$.

No significant associations between the FMS total score and performance tests were recorded in U12, U14, and U16. When analysing associations between the individual FMS subtests, few associations were recorded in the U12 group between TSPU and 
Table 4. Neuromuscular performance (nonadjusted) (mean \pm standard deviation) and FMS individual score (median) in each age category

\begin{tabular}{|c|c|c|c|c|c|c|}
\hline \multirow{2}{*}{ Variable } & \multicolumn{3}{|c|}{ Age groups } & \multicolumn{3}{|c|}{ Hedges g ES } \\
\hline & U12 (n= 15) & U14 (n=14) & U16 (n=12) & U16 vs. U14 & U16 vs. U12 & U14 vs. U12 \\
\hline \multicolumn{7}{|l|}{ Performance } \\
\hline $5 \mathrm{~m}(\mathrm{sec})$ & $1.17 \pm 0.06$ & $1.17 \pm 0.07$ & $1.08 \pm 0.04^{* *, t}$ & 1.50 (large) & 1.67 (large) & 0.06 (small) \\
\hline $10 \mathrm{~m}(\mathrm{sec})$ & $2.04 \pm 0.09$ & $2.00 \pm 0.11$ & $1.85 \pm 0.05^{* *, *}$ & 1.66 (large) & 2.45 (large) & 0.39 (small) \\
\hline 30 m (sec) & $5.28 \pm 0.25$ & $5.05 \pm 0.28$ & $4.43 \pm 0.11^{* *,+}$ & 2.74 (large) & 4.1 (large) & 0.84 (large) \\
\hline 505 COD (non) (sec) & $2.69 \pm 0.16$ & $2.46 \pm 0.09^{* *}$ & $2.36 \pm 0.13^{* *}$ & 0.88 (large) & 2.17 (large) & 1.71 (large) \\
\hline 505 COD (pref) (sec) & $2.66 \pm 0.25$ & $2.43 \pm 0.08^{* *}$ & $2.35 \pm 0.11^{* *}$ & 0.82 (large) & 1.50 (large) & 1.19 (large) \\
\hline CODdef (pref) (sec) & $0.62 \pm 0.24$ & $0.43 \pm 0.13^{*}$ & $0.50 \pm 0.09^{*}$ & 0.60 (moderate) & 0.61 (moderate) & 0.95 (large) \\
\hline CODdef (non) (sec) & $0.65 \pm 0.17$ & $0.46 \pm 0.12^{* *}$ & $0.51 \pm 0.13^{*}$ & 0.39 (small) & 0.88 (large) & 1.51 (large) \\
\hline Y-test (sec) & $2.72 \pm 0.13$ & $2.59 \pm 0.16^{*}$ & $2.22 \pm 0.11^{* * ;+t}$ & 2.57 (large) & 3.99 (large) & 0.87 (large) \\
\hline $\mathrm{CMJ}(\mathrm{cm})$ & $25.11 \pm 4.65$ & $25.06 \pm 3.03$ & $33.37 \pm 3.37^{* *,+}$ & 2.52(large) & 1.94 (large) & 0.01 (small) \\
\hline $\mathrm{SJ}(\mathrm{cm})$ & $25.65 \pm 4.11$ & $24.77 \pm 2.97$ & $33.03 \pm 4.03^{* *,+}$ & 2.29 (large) & 1.76 (large) & 0.42 (small) \\
\hline \multicolumn{7}{|l|}{ FMS score } \\
\hline TS (point) & $15.7 \pm 1.9$ & $16 \pm 1.6$ & $17.2 \pm 1.2$ & & & \\
\hline DS (point) & 2 & 2 & 2 & & & \\
\hline HS (point) & 2 & 2 & 2 & & & \\
\hline ILL (point) & 2 & 2 & 2 & & & \\
\hline SM (point) & 3 & 3 & 3 & & & \\
\hline ASLR (point) & 3 & 2 & 2.5 & & & \\
\hline TSPU (point) & 2 & $3^{*}$ & $3^{* *}$ & & & \\
\hline RS (point) & 2 & 2 & 2 & & & \\
\hline
\end{tabular}

FMS, Functional Movement System; 505 COD (non/pref), 505 change of direction test with nonpreferred/preferred turning leg; CODdef (non/pref), change of direction deficit with nonpreferred/preferred turning leg; Y-test, Y-reactive agility test; CMJ, countermovement vertical jump; SJ, squat jump; TS, total score; DS, deep squat; HS, hurdle step; ILL, in-line lunge; SM, shoulder mobility; ASLR, active straight leg raise; TSPU, trunk stability push-up; RS, rotary stability; ES, effect size.

${ }^{*} P<0.05$ and ${ }^{* *} P<0.01$ compared to the U12. ${ }^{\dagger} P<0.01$ compared to the U14.

5-m sprint time $(r=-0.50, P=0.04, \mathrm{n}=15)$, TSPU and 505COD (preferred) $(r=-0.61, P=0.01, \mathrm{n}=15)$, TSPU and SJ height $(r=$ $0.55, P=0.03, \mathrm{n}=15)$. Interestingly, similar trend was also seen in the U14 between TSPU and CMJ height $(r=0.55, P=0.03$, $\mathrm{n}=14)$, and TSPU and SJ height $(r=0.67, P<0.01, \mathrm{n}=14)$. Contrary and interestingly, in the oldest group U16 significant associations were recorded only between DS and 5-m sprint time $(r=$ $-0.64, P=0.02, \mathrm{n}=12)$, and $\mathrm{DS}$ and $\mathrm{CMJ}$ height $(r=0.58, P=0.04$, $\mathrm{n}=12)$. In the U16, group associations with TSPU and SM could not have been calculated because all the participants achieved the highest possible score.

All the results in each group are shown in Table 4.

\section{DISCUSSION}

The main goal of this study was twofold: firstly, to examine differences between physical characteristics in youth soccer players, and secondly, to find out potential relationships between multiple physical characteristics and the FMS score in youth soccer players of different age categories. Several expected, and few interesting findings were recorded in this study.

First of all, comparison of physical characteristics between all groups showed that in almost every test the oldest group U16 outperformed younger ones (U12 and U14), and U14 outperformed U12 in 5 out of 10 examined physical characteristics. However, these findings were also expected as much as growth and biological maturation plays an important role especially in this age range (Malina et al., 2004; Malina et al., 2015). Regarding acceleration and maximum running speed, an interesting study was performed by Mendez-Villanueva et al. (2011) who compared different age groups of soccer players with significantly better results in favor of the oldest group (U18) compared to the younger ones (U14, U16), however, when the comparison was adjusted for the PHV, all the significant differences disappeared which support the abovementioned statement about growth and maturation as main contributing factors behind the differences between the groups. Abovementioned statements and findings support our results as we have recorded that U16 was significantly quicker compared to the U12 
$(P<0.01)$ and U14 $(P<0.01)$ in $5-, 10-$, and $30-\mathrm{m}$ dash supported by large effect sizes ranged from 0.82 to 4.1. Interestingly, and similarly as in the Mendez-Villanueva et al. (2011) study, we additionally found out significant positive correlations between 5-, $10-$, and 30-m sprint times in each examined age group $(r=0.67-$ $0.89, P<0.01)$ except for the U16 group where the significance level between 5 - and 30-m sprint times was borderline $(r=0.56$, $P=0.055, \mathrm{n}=12$ ). These additional analyses may indicate that acceleration and maximal running speed in youth soccer players may share similar factors which may contradict the findings of professional soccer players (Little and Williams, 2005), and thus, probably different training strategies may be required or in other words, simplicity of linear speed training in youth categories seems to be optimal and should not be intricately separated. However, it should be noted that correlation does not mean causation and thus the results should be interpreted with a caution.

In both variations of the $505 \mathrm{COD}$ test, U16 and U14 were significantly faster compared to the U12 with no significant difference between U16 and U14. This is the only test where no differences were recorded between U16 and U14 and the similar trend was found also in 505 COD deficit (both variations) $(P>0.05)$. In this case, the abovementioned situation may hold also true whereas additional analyses showed positive correlations only in the U16 between 505 COD (preferred) and 5-, 10-, and 30-m sprint time $(r=0.54-0.71, P<0.05)$ as well as 505 COD (nonpreferred) with 5 - and $30-\mathrm{m}$ sprint time $(r=0.52-0.57, P<0.05)$. In the U14 group, only significant correlation was observed between $505 \mathrm{COD}$ (nonpreferred) and $30-\mathrm{m}$ sprint time $(r=0.51, P<0.05)$. No associations were recorded in the U12 group. Abovementioned associations are supported by significant differences obtained between the groups in COD deficit (both variations) which may indicate that not only linear sprinting in the older ones is related to the better performance in COD test but it also seems that technical competence to perform rapid and technically mastered change of direction ability (Nimphius et al., 2016) at an angle of $180^{\circ}$ may be on the higher level in older players. However, it should be noted that even significant but the smallest $P$-values, and small to large effect sizes ranged from 0.39 to 0.88 were recorded when comparing U16 to U12 in the COD 505 deficit in both variations which may indicate reduced ability with greater entry and exit velocities (Loturco et al., 2019). Similarly, as in the 505 COD test, we found that the U16 was significantly quicker in the Y-reactive agility test compared to the U12 and U14 as well. Recorded differences between the groups as mentioned above may be also attributed to the maturation level as described above. Similar results were found in the study by Lloyd et al. (2015) who found that despite the fact that small variation $\left(R^{2}=8 \%\right)$ in reactive agility was recorded, it still suggests that better performance in the U16 group can be explained by higher strength level in relation to the growth and maturation status. Our results in running speed and mainly in pre-planned 505 COD test and Y-reactive agility test could be supported by SJ and CMJ height, whereas the U16 had significantly higher vertical jump height compared to the both younger groups (with large effect sizes ranged from 1.76 to 2.52). However, this may indicate possible higher concentric strength level (Lloyd et al., 2011) that is related to maturation status which can also affect examined outcomes, mainly acceleration, reacceleration as well as cutting skills where rapid force production is essential. It was also shown significant association between $\mathrm{SJ}(r=0.762, P<0.001)$ and $\mathrm{CMJ}(r=0.760, P<0.001)$ height and maximum strength in young soccer players (Comfort et al., 2014).

Interestingly, when results of the performance characteristic were adjusted to the PHV in more than a half of tests significance disappeared (Table 2) which indicate that maturation of the players plays an important role in relation to the physical performance and FMS total score which has been also confirmed in previous studies (Lloyd et al., 2015; Mendez-Villanueva et al., 2011). However, in the study by Lloyd et al. (2015), only reactive strength index remained also unchanged after adjusting to the PHV. Similar results were achieved in our study but in different parameters $(30 \mathrm{~m}$, Y-reactive agility test, $\mathrm{CMJ}$, and $\mathrm{SJ}$ ) which suggests that not only maturation but also probably different training approaches across age categories are responsible for achieved performance differences in youth soccer players.

Regarding the FMS assessment in relation to the physical characteristics we have found few interesting associations but with different trend in each examined group. First, when analysing the FMS total score and all physical characteristics, no significant association in each group was recorded. Similar trend was also recorded in another studies (Parchmann and McBride, 2011; Silva et al., 2017; Zalai et al., 2015) where no association between FMS total score and physical performance was obtained. However, our findings are in contrast to other studies where significant association between FMS total score and physical performance was recorded (Lee et al., 2019; Lloyd et al., 2015). It should be noted that possible discrepancies between the studies may lie in the fact that different physical tests across these studies were performed and importantly, mean total score recorded in our study in each group was still above 15 points which indicate good results and reflect the reality in our age groups, whereas in this case, we were 
not able to divide players according to the low or high score as elsewhere (Lee et al., 2019). And probably due to the abovementioned situation and/or results may be that total score is less sensitive indicator of physical performance. However, when analysing association between individual FMS scores and physical characteristics, several associations in each group were recorded. Interestingly, in the youngest groups (U12), significant associations were recorded between TSPU and $5 \mathrm{~m}$ and 505 COD (preferred) sprint times as well as SJ height. Similar trend was noted in the U14 group between TSPU and CMJ and SJ height. Contrary, in the U16 group, significant associations were obtained between DS and 5-m sprint time and CMJ height. The results in our study when dealing with associations between individual FMS scores and physical performances are in contrast with other studies which examined similar associations with physical performances (Lloyd et al., 2015; Silva et al., 2017). In both mentioned studies, no associations between TSPU and physical performances were recorded. This difference may be caused by different age groups in the study (Silva et al., 2017) or by a statistical approach selected for analysing potential associations (groups joined together) as well as different tests selected (Lloyd et al., 2015). Interestingly, in both younger categories (U12, U14) TSPU was significantly associated to $5 \mathrm{~m}$ and 505 COD sprint times as well as vertical jump height which may indicate different strength status in the groups. Although no significant association was noted in the study by Lloyd et al. (2015), however, they found that TSPU had significant contribution (adjusted $R^{2}=4 \%$ ) in the variation of the reactive strength index which may reflect the importance of trunk muscle stabilization during rapid movements and also change of direction (Sasaki et al., 2011). In addition, when comparing differences between the groups in individual scores, only significant difference was recorded between U16 vs. U12 $(P<0.01)$ and U14 vs. U12 $(P<0.05)$ in TSPU which may indicate (in addition to technical competence) higher strength level of the older groups compared to the younger one. Regarding the association of the DS with 5-m sprint time and $\mathrm{CMJ}$ height in the U16, similar results were recorded in the study by Lloyd et al. (2015), but in this case, it is not clear whether the alike result would be obtained if the group was divided according to the age categories and not grouped together. Significant association in the U16 group may indicate importance of proper execution of the squat which may be related to the better force transmission in short sprint and CMJ height through the kinetic chain.

Regarding FMS as available screening tool, it should be noted that longitudinal assessment of athletes in combination with more sophisticated methods would be necessary to achieve more detailed view or unwanted patterns which could help to provide more accurate and individualized results that are specific and natural for each athlete. As an example, squat technique can be improved by adopting more of a hip hinge strategy to increase range of motion which at the end can be technically optimal execution with respect to individual characteristics of the athletes (Myer et al., 2014).

Limitations of this study include overall number of participants whereas large sample size could validate the results of this study but it is also questionable as limited number of players is available within one age category, and therefore the results obtained in this study are difficult to generalize and are valid for our sample size. Another limitation is that maturation was assessed by prediction equation and not directly, however, it is noninvasive and reliable method to evaluate maturation in youth athletes.

In conclusion, the results of this study suggest that physical performance in youth soccer players is a consequence of a wide range of factors including maturation, various training methods across age categories (e.g., older players may undergo more intense training strategies compared to younger ones) and movement proficiency. All of that (including endurance, strength, speed, mobility, and agility training) should be rationally developed throughout childhood and adolescence that may lead to balanced development of an individual with a perspective to future success.

\section{CONFLICT OF INTEREST}

No potential conflict of interest relevant to this article was reported.

\section{REFERENCES}

Bardenett SMM, DeNoyelles JT, Miller SD, Jenk DT, Brooks GS. Functional movement screen normative values and validity in high school athletes: Can the fms be used as a predictor of injury? Int J Sports Phys Ther 2015;10:303-308.

Behm DG, Faigenbaum AD, Falk B, Klentrou P. Canadian Society for Exercise Physiology position paper: resistance training in children and adolescents. Appl Physiol Nutr Metab 2008;33:547-561.

Comfort P, Stewart A, Bloom L, Clarkson B. Relationships between strength, sprint, and jump performance in well-trained youth soccer players. J Strength Cond Res 2014;28:173-177.

Cook G. Movement: functional movement systems. Screening-assessmentcorrective strategies. Chichester (UK): Lotus Publishing; 2011.

Dugdale JH, Sanders D, Hunter AM. Reliability of change of direction 
and agility assessments in youth soccer players. Sports (Basel) 2020; 8:51.

Figueiredo AJ, Gonçalves CE, Coelho E Silva MJ, Malina RM. Youth soccer players, 11-14 years: maturity, size, function, skill and goal orientation. Ann Hum Biol 2009;36:60-73.

Hopkins WG. A scale of magnitudes for effect statistics [Internet]. Will G Hopkins; 2016 [cited 2020 Jun 3]. Available from: https://www.sportsci.org/resource/stats/effectmag.html.

Kiesel K, Plisky PJ, Voight ML. Can serious injury in professional football be predicted by a preseason functional movement screen. $\mathrm{N} \mathrm{Am} \mathrm{J}$ Sports Phys Ther 2007;2:147-158.

Kobal R, Pereira LA, Zanetti V, Ramirez-Campillo R, Loturco I. Effects of unloaded vs. loaded plyometrics on speed and power performance of elite young soccer players. Front Physiol 2017;26:742.

Lee S, Kim H, Kim J. The Functional Movement Screen total score and physical performance in elite male collegiate soccer players. J Exerc Rehabil 2019;15:657-662.

Letafatkar A, Hadadnezhad M, Shojaedin S, Mohamadi E. Relationship between functional movement screening score and history of injury. Int J Sports Phys Ther 2014;9:21-27.

Little T, Williams AG. Specificity of acceleration, maximum speed, and agility in professional soccer players. J Strength Cond Res 2005;19: 76-78.

Lloyd RS, Oliver JL. The youth physical development model: a new approach to long-term athletic development. Strength Cond J 2012;34: 61-72.

Lloyd RS, Oliver JL, Hughes MG, Williams CA. Specificity of test selection for the appropriate assessment of different measures of stretch-shortening cycle function in children. J Sports Med Phys Fitness 2011;51: 595-602.

Lloyd RS, Oliver JL, Radnor JM, Rhodes BC, Faigenbaum AD, Myer GD. Relationships between functional movement screen scores, maturation and physical performance in young soccer players. J Sports Sci 2015;33:11-19.

Lockie R, Schultz A, Callaghan S, Jordan C, Luczo T, Jeffriess M. A preliminary investigation into the relationship between functional movement screen scores and athletic physical performance in female team sport athletes. Biol Sport 2015;32:41-51.

Loturco I, Pereira LA, Freitas TT, Alcaraz PE, Zanetti V, Bishop C, Jeffreys I. Maximum acceleration performance of professional soccer players in linear sprints: is there a direct connection with change-of-direction ability? PLoS One 2019;14:e0216806.

Loturco I, Pereira LA, Kobal R, Maldonado T, Piazzi AF, Bottino A, Kitamura K, Cal Abad CC, de Arruda M, Nakamura FY. Improving sprint performance in soccer: effectiveness of jump squat and Olympic push press exercises. PLoS One 2016;11:e0153958.

Malina RM, Eisenmann JC, Cumming SP, Ribeiro B, Baros J. Maturity associated variation in the growth and functional capacities of youth football (soccer) players 13-15 years. Eur J Appl Physiol 2004;91:555562.

Malina RM, Rogol AD, Cumming SP, Coelho e Silva MJ, Figueiredo AJ. Biological maturation of youth athletes: assessment and implications. Br J Sports Med 2015;49:852-859.

Marques VB, Medeiros TM, de Souza Stigger F, Nakamura FY, Baroni BM. The functional movement screen (FMS ${ }^{\mathrm{TM}}$ ) in elite young soccer players between 14 and 20 years: composite score, individual-test scores and asymmetries. Int J Sports Phys Ther 2017;12:977-985.

McCall AC, Davison M, Nedelec M, Le Gall F, Berthoin S, Dupont G. Injury risk factors, screening tests and preventative strategies: a systematic review of the evidence that underpins the perceptions and practices of 44 football (soccer) teams from various premier leagues. Br J Sports Med 2015;49:1-8.

Mendez-Villanueva A, Buchheit M, Kuitunen S, Douglas A, Peltola E, Bourdon P. Age-related differences in acceleration, maximum running speed, and repeated-sprint performance in young soccer players. J Sports Sci 2011;29:477-484.

Mirwald RL, Baxter-Jones ADG, Bailey DA, Beunen GP. An assessment of maturity from anthropometric measurements. Med Sci Sports Exerc 2002;34:689-694.

Myer GD, Kushner AM, Brent JL, Schoenfeld BJ, Hugentobler J, Lloyd RS, Vermeil A, Chu DA, Harbin J, McGill SM. The back squat: a proposed assessment of functional deficits and technical factors that limit performance. Strength Cond J 2014;36:4-27.

Newton F, McCall A, Ryan D, Blackburne C, Fünten K, Meyer T, Lewin C, McCunn R. Functional Movement Screen (FMS ${ }^{\mathrm{TM}}$ ) score does not predict injury in English Premier League youth academy football players. Sci Med Football 2017;1:102-106.

Nimphius S, Callaghan SJ, Spiteri T, Lockie RG. Change of direction deficit: a more isolated measure of change of direction performance than total 505 time. J Strength Cond Res 2016;30:3024-3032.

Parchmann CJ, McBride JM. Relationship between functional movement screen and athletic performance. J Strength Cond Res 2011;25:33783384.

Sasaki S, Nagano Y, Kaneko S, Sakurai T, Fukubayashi T. The relationship between performance and trunk movement during change of direction. J Sports Sci Med 2011;10:112-118.

Shultz R, Anderson SC, Matheson GO, Marcello B, Besier T. Test-retest and interrater reliability of the functional movement screen. J Athl Train 2013;48:331-336.

Silva B, Clemente FM, Camões M, Bezerra P. Functional movement screen 
scores and physical performance among youth elite soccer players. Sports (Basel) 2017;5:16.

Smith PD, Hanlon MP. Assessing the effectiveness of the functional movement screen in predicting noncontact injury rates in soccer players. J
Strength Cond Res 2017;31:3327-3332.

Zalai D, Bobak P, Csaki I, Hamar P, Myrer JW, Mitchell UH, Johnson AW. Motor skills, anthropometrical characteristics and functional movement in elite young soccer players. J Exerc Sports Orthop 2015;2:1-7. 